\title{
Accidental Poisoning in Children - At Tertiary Care Unit
}

\author{
Dr.B.Narahari ${ }^{1}$,Dr.C.Chandramohan ${ }^{2}$,Dr.M.Ranjeet ${ }^{3}$,Dr. K. Sreenivas ${ }^{4}$ \\ ${ }^{1,2}$ Assistant Professor of Pediatrics, Department of Pediatrics, Osmania Medical College, Hyderabad. \\ ${ }^{3}$ Senior Resident of Pediatrics, Department of Pediatrics, Osmania Medical College, Hyderabad. \\ ${ }^{4}$ CAS, Niloufer Hospital, Osmania Medical College, Hyderabad. \\ Corresponding Author: Dr.C.Chandramohan,
}

\begin{abstract}
:
Background: Poisoning is a very common emergency in the pediatric department which causes a lot of worry and anxiety to parents as well as doctors. The incidence type and nature of poisoning is influenced by various factors hence a study was proposed on poisoning to form treatment protocols.

Aims and Objectives: To conduct a study on Accidental poisoning in children and to find out commonest poisoning, its route, type of poison with clinical features, mortality, and outcome of cases of accidental poisoning. Study design: Prospective observational study of all cases of poisoning.

Material And Methods: Hundred children who were below 12 years of age who had accidental poisoning admitted in pediatric emergency department at Niloufer Hospital were taken for study from 2016 November to 2017 June. Results: out of 100 cases studied oral route (89\%) parenteral route (11\%), age distribution below 5 years were (67.6\%), urban cases (71\%), rural cases (29\%), time since poisoning within 6hours( $81 \%$ ), after 6 hours of exposure(19\%), commonest clinical features involved is vomiting (40\%), kerosene was commonest type of poisoning with (44\%), mortality was (2\%).

Conclusions: The oral route of poisoning was more common than parenteral route, commonest age group was toddlers, most of the patients were from urban population, most common poison being kerosene. The time taken to transfer patient from rural area was twice that of urban area, duration of stay for most patients was between 1 to 2 days, mortality in the study was (2\%).
\end{abstract}

Keywords: Accidental poisoning, Kerosene poisoning, Poisoning, Route, Urban, Rural.

\section{Introduction}

Poisoning is a very common emergency in the pediatric department which causes a lot of worry and anxiety to parents as well as doctors. The incidence type and nature of poisoning vary from region to region and is influenced by lifestyle, socioeconomic status and cultural habits therefore it is necessary to study poisoning at different times in order to formulate proper treatment protocols. References to poisons are found in the oldest Egyptian Babylonian and Greek records. Susrutha has described several modes of poisoning ${ }^{1}$.The medico legal aspects of Poison Act 1919 which regulates the import, possession poisons and sale of poisons. Drugs and Cosmetics Act 1940 regulates manufacture and sale of drugs. Drugs control Act 1950 empowers the producer and dealer to fix maximum price of any drug .Various Sections 284,299,300,304A, 324,326,328 IPC deal with offences related to administration of poisoning ${ }^{1}$.

Accidental poisoning in children is a global problem ${ }^{2}$ and it is the twelfth leading cause of admissions to hospital in India. Most cases of accidental poisoning are preventable. As children start crawling walking they become very active try to explore unfamiliar objects putting them in mouth and tasting. Poisons can be classified $^{1}$ into Corrosives, opiods, dathura, botulism, household poisons like nail polish remover (acetone), Hydrocarbons like kerosene, turpentine oil. Agricultural pesticides like organophosphorus, drugs like asprin, iron, and antiepileptic. Snake and scorpion envenomations. The Routes of administration of accidental poisoning can be Inhalational, ingestion, injection, contact, introducing into orifices rectum and vagina. Factors modifying the action of poisons include the quantity and route of administration. High index of suspicion plays a key role like, sudden appearance of symptoms in a previously healthy child. Certain preventive measures can well be useful like, all the chemicals drugs and potentially toxic poisons should be kept out of reach of children. Hydrocarbons like kerosene should not be stored in cool drink bottles rather to be stored in original packing only. Avoid taking medicines in presence of children because they love to imitate our actions. All parents should be educated regarding poisonous nature of household substances. The address and phone numbers of the nearest hospital should be easily available, Doctors are the best source of medical information ${ }^{1}$. Legislation is required for establishment of poison information centers which provide information about type of poison and treatment over phone and also store antidotes for the common poisons. 


\section{Material And Methods}

A prospective observational study of all cases of poisoning was designed and study setting included 100 children less than 12 years who were brought to pediatric emergency department during the period November 2016 to June 2017.Inclusion criteria: Children who had accidental poisoning less than 12 years of age. Exclusion criteria: Children more than 12 years of age. Those with allergic reactions to plants. Those with allergic reactions to foods. Those with food poisoning due to infective causes. Data collected was formated and analyzed using SPSS for windows version 1.0 Observations were done regarding the route of poisoning, sex distribution, age wise distribution, area wise distribution, type of poison, interval between poisoning and admission, duration of stay in hospital, clinical features and a special emphasis has been laid on kerosene poisoning because it is the commonest type of poisoning in this study.

\section{Results}

Out 100 children studied the oral route of poisoning was observed in $89 \%$ of children and parenteral route in $11 \%$ of children.

FIG 1: Route Of Poisoning

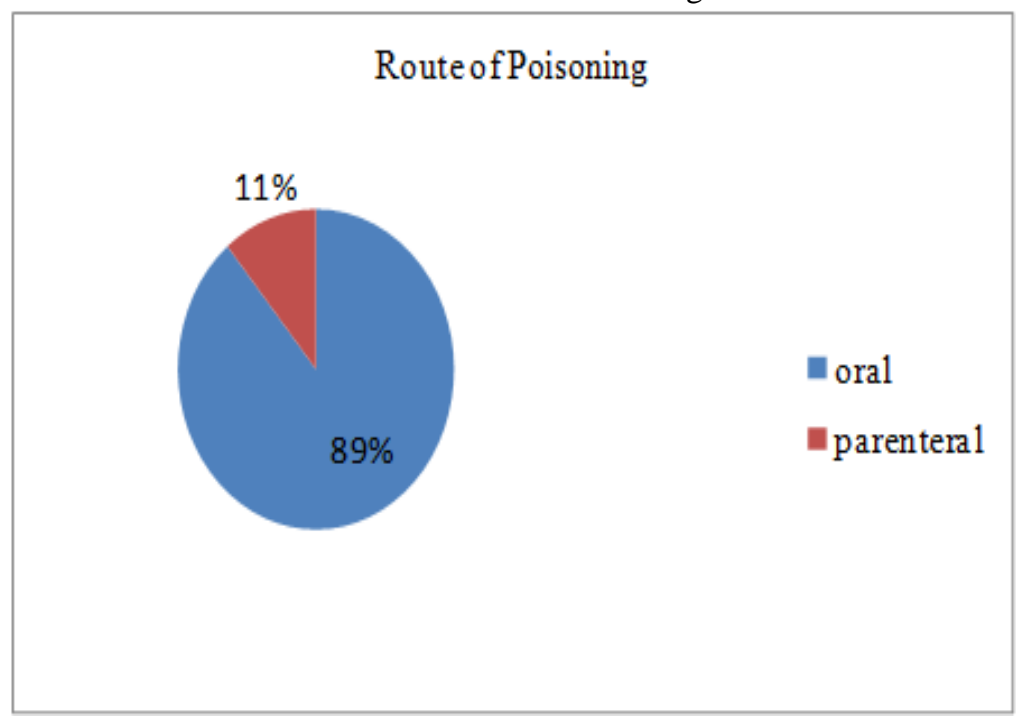

Fig 2 : Sex Wise Distribution

In our study male to female ratio was $1.3: 1$

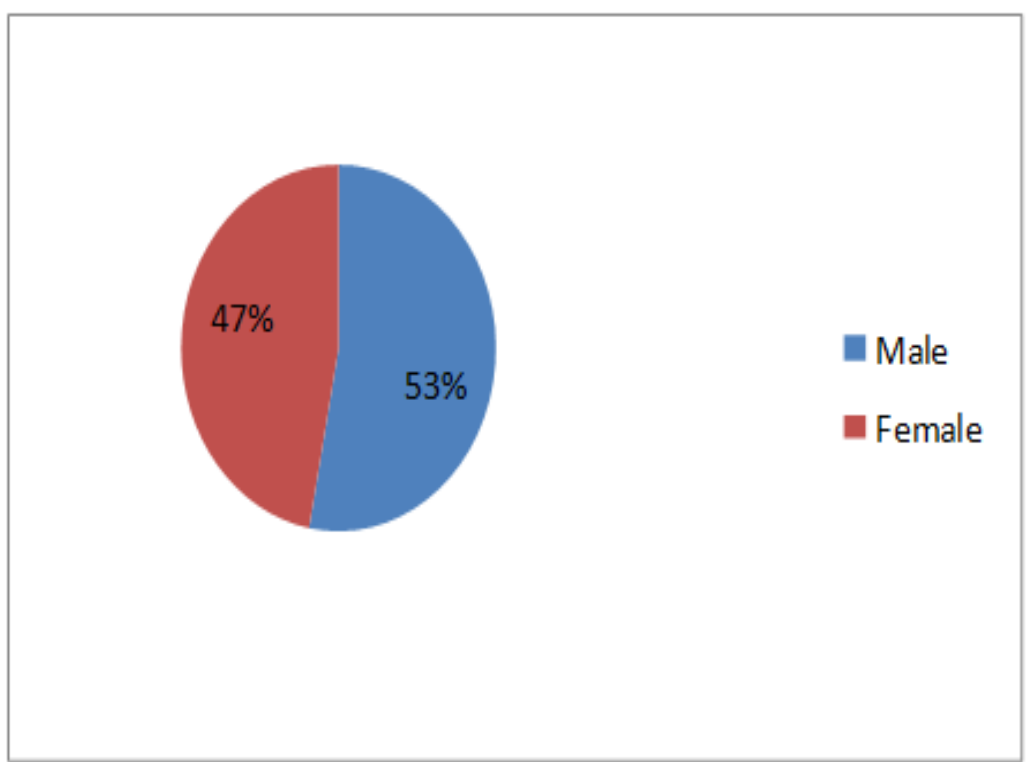

FIG 2 : Sex Wise Distribution 
In our study male to female ratio was $1.3: 1$

Table 1: Age Wise Distribution

\begin{tabular}{|l|l|l|}
\hline Age & No. of cases & Percentage \\
\hline$<1$ year(infant) & 5 & 5 \\
\hline 1-3 years & 68 & 68 \\
\hline 3-5years & 12 & 12 \\
\hline 5-10 years & 14 & 14 \\
\hline $10-12$ years & 1 & 1 \\
\hline
\end{tabular}

The commonest age group involved in poisoning was1-3 year's toddlers (68).

Fig 3: Area Wise Distribution

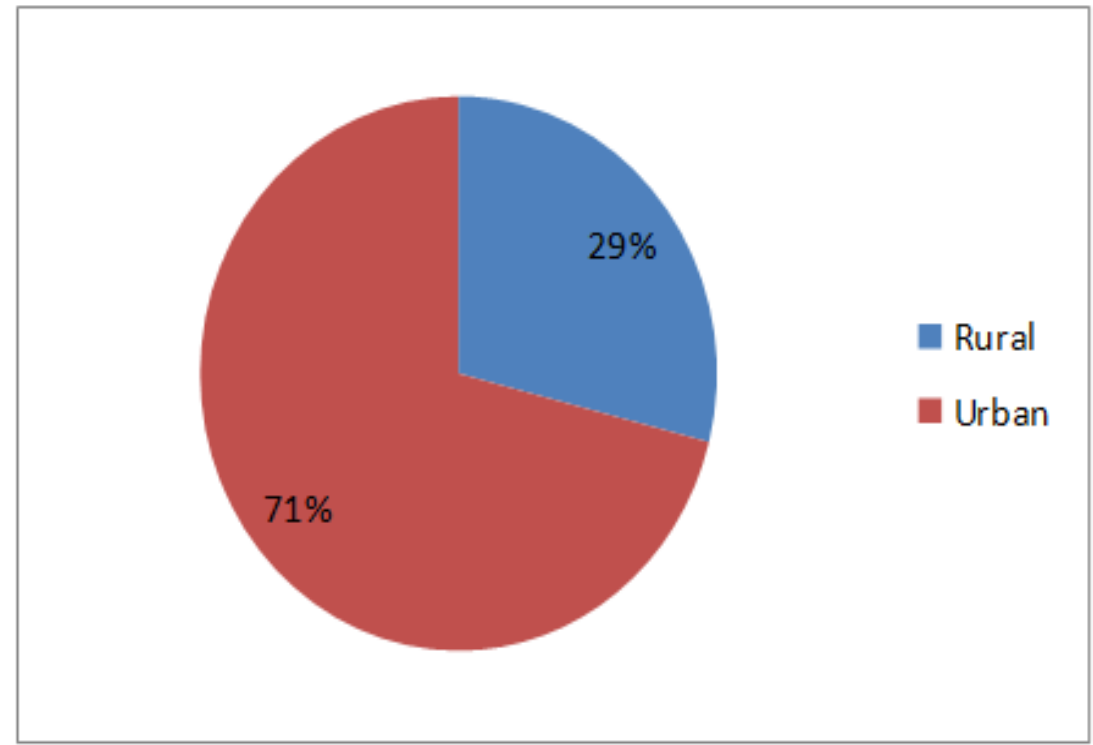

In our study More number of cases were from Urban area(71\%).

Table 2: Category (Type) Of Poisoning

\begin{tabular}{|l|l|l|l|}
\hline S1.NO. & Category & No. of cases & Percentage \\
\hline 1. & Hydrocarbons & 57 & 57 \\
\hline 2. & Household chemicals & 13 & 13 \\
\hline 3. & Drugs & 8 & 8 \\
\hline 4. & Snake bites & 7 & 7 \\
\hline 5. & Agrochemicals & 7 & 7 \\
\hline 6. & Scorpion bite & 4 & 4 \\
\hline 7. & unknown & 4 & 4 \\
\hline
\end{tabular}

Among the Hydrocarbon poisoning kerosene was most common type of poisoning in this study followed by ingestion of Household chemicals. Among the parenteral type of poising snake bites were common.

Table 3: Interval Between Poisoning And Admission

\begin{tabular}{|l|l|l|}
\hline Time & No. of cases & percentage \\
\hline Up to 6 hours & 81 & 81 \\
\hline$>$ 6hours & 19 & 19 \\
\hline
\end{tabular}

In our study $81 \%$ of cases were brought within 6 hours of poisoning.

Table 4: Kerosene Poisoning

\begin{tabular}{|l|l|l|l|}
\hline Sl.NO. & System involved & No. of cases & Percentage \\
\hline 1 & Respiratory system & 30 & 68 \\
\hline 2 & Gastrointestinal system & 10 & 23 \\
\hline 3 & Central nervous system & 2 & 5 \\
\hline
\end{tabular}

Among the kerosene poisoning cases considered for study the most common system involved was Respiratory system followed by gastrointestinal system. 
Table 5: Kerosene Poisoning Symptoms

\begin{tabular}{|l|l|l|}
\hline Symptoms & No. of cases & Percentage \% \\
\hline Breathlessness & 23 & 52 \\
\hline Cough & 19 & 43 \\
\hline Vomiting & 10 & 22 \\
\hline Fever & 10 & 22 \\
\hline Irritability & 10 & 22 \\
\hline Abdominal pain & 8 & 18 \\
\hline Altered sensorium & 2 & 4 \\
\hline Abdominal distension & 2 & 4 \\
\hline seizures & 2 & 4 \\
\hline
\end{tabular}

In our study the commonest symptom in kerosene poisoning was Breathlessness followed by cough, vomiting, fever, irritability.

Table 6: Kerosene Poisoning Signs

\begin{tabular}{|l|l|l|}
\hline Sign & cases & Percentage \% \\
\hline Crepitations & 23 & 52 \\
\hline Tachypnea & 23 & 52 \\
\hline Tachycardia & 12 & 27 \\
\hline Retractions & 7 & 15 \\
\hline cyanosis & 2 & 4 \\
\hline
\end{tabular}

In our study the commonest sign in kerosene poisoning was crepitations, followed by tachypnoea, tachycardia.

Table 7: Radiological Appearance In Kerosene Poisoning

\begin{tabular}{|l|l|l|}
\hline Chest X ray appearance & No. of cases & Percentage \% \\
\hline Normal & 22 & 50 \\
\hline Right Para cardiac pneumonitis & 16 & 36.5 \\
\hline Bilateral pneumonitis & 4 & 9 \\
\hline White out lung & 2 & 4.5 \\
\hline
\end{tabular}

In our study the commonest finding in chest $\mathrm{x}$ ray was normal study, followed by right para cardiac pneumonitis.

\section{Discussion}

Out of 100 cases of poisoning the oral route accounted for $89 \%$. Whereas the parenteral route accounted for $11 \%$. In a study by Khadgawat et al. ${ }^{3}$., the incidence of oral route of poisons was $66.7 \%^{3}$. Other routes of poisoning have been demonstrated by Josef $\mathrm{H}$ et al, ${ }^{4}$ (Trans conjunctival atropine ointment toxicity) and soni et al, ${ }^{5}$ (Inhaled mercury vapour). The male to female ratio in our study was $1.3: 1$, in similar studies on poisoning done by Buch et.al ${ }^{6}$, Kumar et.al ${ }^{7}$. Singh et al $^{8}$. The male to female ratio were 1.25:1 and 2:1 and 3.1:1 respectively. In our study, incidence of poisoning was seen in 1 to 3 years age group (68), 3 to 5 years age group (12). Only 5 were infants and least affected were pre pubertal adolescent (1). This finding is similar to study done by Khadgawat et $\mathrm{al}^{3}$ where two thirds were below 5 years. In study done by Sharma et $\mathrm{al}^{9}$ it was found that intrauterine babies can be poisoned by transplacental transfer of carbamate and in another study done by dev and chaturvedi et al. ${ }^{10}$ children as young as 7 months have been affected.

In our study the urban cases reported were $71 \%$ and rural were $29 \%$. In the study done by Sharma et al

${ }^{11}$ had more urban children with urban to rural ratio of 1.13:1 was noted. In study done by Kumar et al, . ${ }^{7}$ had more from children rural areas with ratio of rural to urban of 5:2.In our study the parenteral poisonings constituted $11 \%$ commonest was snake bite. In the study by Khadgawat et al ${ }^{3}, 15 \%$ of all snake bites were due to non poisonous snakes. In the studies done by kulkarni et $\mathrm{al}^{12}$ and agarwal et $\mathrm{al}^{13}$ they have concentrated only on snake poisoning. In our study $81 \%$ children were brought to hospital within 6 hours and $19 \%$ were brought after 6 hours. This has a significant bearing on the outcome of the poisoning. In our study $87 \%$ children were discharged within 2 days. In a similar study by gupta et al. ${ }^{14}$ the duration of hospital stay was $48 \mathrm{hrs}$.

Manifestations Of Kerosene Poisoning: Though most of the kerosene poisoning occurs by the oral route, it can also be absorbed through skin, as studied by sarkar et al. ${ }^{15}$. In our study the commonest symptom was breathlessness $(52 \%)$ followed by cough(43\%)and vomiting(22\%) and fever (22\%). The commonest sign was crepitations $(52 \%)$ followed by tachypnea $(23 \%)$. (In study by Gupta et al. ${ }^{16}$ the findings were in order of frequency of breathlessness(55.7\%), fever(47.1\%)retractions(35.7\%), cough (31.4\%)and restlessness $(25.7 \%)$.

In our study 22 children had normal chest xrays, 16 children had Right Para cardiac pneumonitis 4 children had bilateral pneumonitis and 2 children had white out lungs. In study conducted by Khadgawat et al, ${ }^{3}$ chest $\mathrm{x}$ rays were showing pneumonitis in $40.3 \%$ of patients. In our study the mortality was $2 \%$ and the cause noted was respiratory failure. In study done by Dutta et al ${ }^{16}$ the mortality ranged from 0.64 to $11.6 \%$. 


\section{Conclusion}

The oral route of poisoning was more common than parenteral route, commonest age group was toddlers, and most of the patients were from urban population, most common poison being kerosene. The time taken to transfer patient from rural area was twice that of urban area, duration of stay for most patients was between 1 to 2 days, mortality in the study was (2\%).

\section{Acknowledgements}

The authors are grateful to the emergency department, parents and the guardians who consented for the participation in the study.

\section{References}

[1]. Reddy K.S.N, Essentials of Forensic Medicine and Toxicology $26^{\text {th }}$ edition 2007.

[2]. Ghai.o.p.-Essential Pediatrics; 6th edition; 2006.

[3]. Khadgawat P.,GargP.,Bansal P,Arya A.,Choudhary B.-Accidental Poisoning; Indian Pediatrics.,Vol.31;December.1994;PP.15551557.

[4]. Joseph V.J.; Rekha S.; Subba rao S.D.; Chandreashekhar M.K.; An unusual case of atropine toxicity; Indian Pediatrics; Vol.28;August 1991; PP.943-944

[5]. Sony J.P.;Singhani R.V.,Bansal A., Rathi G-Acute Mercury vapor poisoning ; Indian Pediatrics;Vol;March 1992;PP 365-368.

[6]. Buch N.A., Ahmed K., Sethi A.S.,-Poisioning in Children; Indian Pediatrics; Vol 28; May1991; PP.521-524.

[7]. Kumar V.,-Accidental Poisioning in South West Maharashtra; Indian pediatrics; Vol.28; July 1998; PP 731-734.

[8]. Singh R.P.; Loperamide poisoning in children; Indian Pediatrics; Vol.28; May 1991; PP578.

[9]. Sharma A., Oral Aluminum phosphide poisoning, Indian Pediatrics; Vol. 32; March1995; PP $339-341$.

[10]. Dey S.K., chaturvedi P., Jain V,-Street heroin poisoning in a seven month old infant; Indian Pediatrics; Vol.29;March 1992.

[11]. Sharma A., Oral Aluminum phosphide poisoning, Indian Pediatrics; Vol. 32; March1995; PP 339-341.

[12]. Kulkarni M.L.; Anees S.-Snake venom poisioning, Experience with 633 cases-Indian Pediatrics; Vol.31; October 1994, PP.12391242.

[13]. Aggarwal R., Thavaraj V., Snake Envenomations, Indian Pediatrics Vol.31, October 1994, P 1309-1312.

[14]. Gupta P. Singh R.P., Murali M.V.; Sharma P.P.,Prognostic score for kerosene oil poisoning; Indian Pediatrics;Vol.29;August 1992; PP.1109-1112.

[15]. Sarkar A.K., SarkarM., Biswas S.,-An Unusual case of kerosene oil poisoning; Indian Pediatrics; Vol.31; January 1994,PP67-68.

[16]. Dutta A.K.et at; poisoning in children-Indian Scenario; Indian Journal of Pediatrics; vol.65; May-June1998-PP.365-370. 